\title{
Treatment with Dalteparin is Associated with a Lower Risk of Bleeding Compared to Treatment with Unfractionated Heparin in Patients with Renal Insufficiency
}

\author{
Doyun Park, $M D^{7}$, William Southern, MD, MS², Manuela Calvo, MD², Margarita Kushnir, MD', \\ Clemencia Solorzano, $\mathrm{RPH}^{4}$, Mark Sinnet, PharmD ${ }^{4}$, and Henny H. Billett, MD, MSc ${ }^{3}$
}

'Division of Hematology-Oncology, Mount Sinai-Roosevelt Hospital, New York, NY, USA; ${ }^{2}$ Division of Hospital Medicine, Department of Medicine, Montefiore Medical Center of Albert Einstein College of Medicine, Bronx, NY, USA; ${ }^{3}$ Division of Hematology, Department of Oncology, Montefiore Medical Center of the Albert Einstein College of Medicine, Bronx, NY, USA; ${ }^{4}$ Department of Pharmacy, Montefiore Medical Center, Bronx, NY, USA.

BACKGROUND: Low molecular weight heparins (LMWHs) have been cautiously used in patients with chronic kidney disease (CKD) due to fear of accumulation. Dalteparin, however, has shown minimal tendency to accumulate in patients with CKD and may be safe to use in this patient population.

OBJECTIVE: We compared the incidence of clinically significant bleeding in patients with $\mathrm{CKD}$ receiving therapeutic doses of dalteparin to that of patients with CKD receiving therapeutic doses of UFH.

DESIGN: This was a retrospective cohort study.

SUBJECTS: Inpatients with CKD (GFR<60 ml/min) who were treated with therapeutic dalteparin or UFH were included in the study

MAIN MEASURES: Primary outcome was major bleeding within 10 days of anticoagulation, identified by ICD-9 code and confirmed by chart review. Demographic characteristics, laboratory values, comorbidities, prior bleeding history and inpatient medications were extracted for each admission from the electronic medical record. Logistic regression models were created to examine the association between choice of anticoagulant and bleeding rates, after adjustment for demographic and clinical characteristics.

KEY RESULTS: Dalteparin-treated patients were significantly less likely to experience a major bleed than patients treated with UFH (1.14 \% vs. $3.49 \%, p<0.001)$. The reduced likelihood of bleeding associated with dalteparin treatment remained significant after adjustment for patient characteristics (HR 0.39, $95 \%$ CI: 0.21-0.70, $p<0.0001)$. A stratified analysis for subgroups with GFR $<30 \mathrm{~mL} / \mathrm{min}$ and with GFR between 30 and $60 \mathrm{~mL} / \mathrm{min}$ showed that dalteparin was still associated with lower odds of bleeding compared to treatment with unfractionated heparin, but the difference was nonsignificant for GFR< 30 (HR 0.35, 95 \% CI: 0.11-1.15), even after adjustment (OR 0.37, $95 \%$ CI: 0.11-1.22).

CONCLUSION: In patients with CKD, treatment with therapeutic dose dalteparin was associated with lower rates of bleeding than treatment with unfractionated heparin. For patients with severe CKD (GFR<30), dalteparin

Received January 6, 2015

Revised June 5, 2015

Accepted June 29, 2015

Published online July 25, 2015 was shown to be at least as safe as unfractionated heparin.

KEY WORDS: dalteparin; renal insufficiency; bleed; heparin.

J Gen Intern Med 31(2): 182-7

DOI: $10.1007 / \mathrm{s} 11606-015-3466-4$

(C) Society of General Internal Medicine 2015

\section{INTRODUCTION}

Low molecular weight heparins (LMWH) have come into prominence for their ease of use, superior bioavailability and more predictable pharmacokinetics in comparison to unfractionated heparin. ${ }^{1,2}$ Unlike unfractionated heparins (UFH), LMWHs have less binding to plasma proteins and thus have more predictable anticoagulant activities, which allow them to be given as daily subcutaneous injections without monitoring under most circumstances. ${ }^{3}$ Multiple studies have demonstrated LMWHs to be as effective an anticoagulant, and in some populations even more efficacious, than unfractionated heparin or the vitamin $\mathrm{K}$ antagonist warfarin, with an equal or better bleeding profile. ${ }^{4,5}$ Because LMWHs are primarily excreted by the kidney, there is concern for bioaccumulation of LMWHs in patients with chronic renal insufficiency receiving repeated doses, in particular with the hydrophilic enoxaparin. ${ }^{6}$

However, given the varying molecular and pharmacokinetic features of the LMWHs, results found with one type of LMWH are not necessary applicable to other LMWHs. Sigueret, Pautas, and their group showed that there was no accumulation of anti-factor Xa activity over a 10-day treatment period of therapeutic tinzaparin, and in a subsequent study conducted over 30 days, reported the absence of correlation between anti-Xa levels and creatinine clearance with therapeutic tinzaparin therapy. ${ }^{7,8}$ Several studies using dalteparin have suggested that bioaccumulation does not occur with prophylactic doses. ${ }^{9}$ A single study comparing 4-h postinjection anti-Xa levels in patients with a mean glomerular filtration rate (GFR) of 26.1 (range 16-38) $\mathrm{ml} / \mathrm{min}$ versus normal renal function after therapeutic doses of dalteparin did not show any difference in the assay levels in those with 
impaired renal function when compared to those with normal renal function. ${ }^{10}$

Currently, little is known about bleeding risks with therapeutic doses of dalteparin in patients with renal insufficiency, and guidelines regarding dose adjustments and preference of unfractionated heparin over dalteparin in this population are not sufficiently evidence-based. ${ }^{11}$ To our knowledge, there has not been a prospective or retrospective study in which the bleeding risk of therapeutic dose of dalteparin in patients with renal insufficiency has been examined. Our objective was to compare the incidence of clinically significant bleeding in patients with renal insufficiency after having received therapeutic doses of dalteparin vs. UFH. We hypothesized that the dalteparin cohort would not be associated with increased bleeding rates compared to the UFH cohort.

\section{METHODS}

Trial Design and Participants. We conducted a single center, retrospective study consisting of patients who were admitted at Montefiore Medical Center between 1 January 2006 and 30 June 2010 using a clinical analytics software tool called Clinical Looking Glass (CLG), an interactive software application developed at Montefiore Medical Center to evaluate health care quality, effectiveness, and efficiency. The system integrates clinical and administrative data sets, enabling the extraction of cross-sectional and longitudinal data suitable for epidemiological analyses. We identified eligible inpatients who were 18 years or older who received either therapeutic doses of dalteparin or intravenous heparin for clinical indications including venous or arterial thromboembolism (VTE), atrial fibrillation and bridging prior to surgery, and myocardial infarction. Therapeutic dosing was defined as documentation of at least 10,000 units daily - minimum VTE treatment dose for weight $<57 \mathrm{~kg}^{12}$ - given for a minimum of 3 days, or unfractionated heparin given for at least 3 days with documentation of aPTT $>50 \mathrm{~s}$. Renal impairment was defined by GFR, derived from Modification of Diet in Renal Disease (MDRD) equations, with cutoff levels of $30 \mathrm{ml} / \mathrm{min}$ and $60 \mathrm{ml} /$ min used for our subgroup analyses. After the initial data retrieval, individual charts and/or electronic medical records were retrieved and reviewed to ensure they met eligibility criteria and to assess outcomes. Patients who had unstable creatinine levels, defined as a rise of $>50 \%$ from the initial creatinine to the highest creatinine during a single admission, were excluded. Those who did not meet the dosage/duration requirement or who were receiving concomitant therapeutic warfarin with INR $\geq 2$ were also excluded, as were patients with platelet counts $<50 \times 10^{9} / \mathrm{L}$.

Outcome measures and statistical analysis. The primary analysis was the incidence of clinically significant (major) bleeding events that occurred within 10 days of the first dose of therapeutic anticoagulation. Diagnostic criteria for clinically significant and/or major bleeding were adopted from those recommended by the Control of Anticoagulation Subcommittee: 1) fatal bleed; 2) symptomatic bleed at anatomically critical sites such as intracranial, intraspinal, intraocular, or pericardial; 3) symptomatic noncritical bleeds resulting in transfusion of 2 units or more of red blood cells or drop in hemoglobin of at least $2.0 \mathrm{~g} / \mathrm{dl}$, or need for surgical re-intervention. ${ }^{13}$ Bleeding events could either be during the admission or after discharge, and could be on or off treatment. Events were captured by chart review of all admission, discharge, progress and operative notes. Any blood product administration was also recorded. For sample size calculation, we designated an absolute difference of $1 \%$ in bleeding rate to be clinically significant. Baseline characteristics were summarized per intervention group using mean and standard deviation, or percentages. Characteristics of patient groups were compared using t-tests, and chi-square tests as appropriate. Hazard ratios $(95 \% \mathrm{CI})$ were calculated to determine the relative effect of the two anticoagulants on the primary outcome.

Time from the start of treatment to first episode of major bleed was assessed using the methods of Kaplan and Meier, including separate analyses for bleeding events censored at 10 days and 30 days after the start of treatment. Differences in bleeding between patient groups were assessed using log-rank tests. To determine the independent association between the use of unfractionated heparins vs. dalteparin and the risk of bleeding, we constructed Cox-proportional hazards models with 10-day bleeding as the outcome. Patient characteristics, including age, sex, Charlson score, history of bleeding, use of warfarin, platelet count, GFR and INR, were included in the models. Separate proportional hazards models were constructed for patients with GFR $<30 \mathrm{ml} / \mathrm{min}$ and GFR $30-60 \mathrm{ml} / \mathrm{min}$. STAT/IC version 13.1 was used for all statistical analyses.

\section{RESULTS}

Study Population. Demographic and clinical characteristics of the study population are presented in Table 1 . We identified 3,186 patients admitted to the medical service from 1 January 2006 to 30 June 2010 with GFR $<60 \mathrm{ml} / \mathrm{min}$ who were treated with therapeutic doses of either dalteparin or UFH. Of these, $1,321(41.5 \%)$ were treated with dalteparin and 1,865 $(58.5 \%)$ were treated with UFH. Patients treated with dalteparin were older (mean age 72.3 vs. 70.5 years), more likely to be female (61.4\% vs $48.3 \%$ ), and had a higher GFR (42.8. vs. $34.8 \mathrm{~mL} / \mathrm{min}$ ) and fewer comorbidities (mean Charlson score 3.1 vs. 3.7) compared to patients treated with UFH. Both patient groups were racially and ethnically diverse with no significant differences in racial/ethnic characteristics. 
Table 1. Patient Demographics and Clinical Characteristics by Type of Anticoagulation Received (Dalteparin vs. Unfractioned Heparin)

\begin{tabular}{|c|c|c|c|}
\hline & \multirow{2}{*}{$\begin{array}{l}\text { Dalteparin } \\
(n=1321)\end{array}$} & \multirow{2}{*}{$\frac{\text { UFH }}{(n=1865)}$} & \multirow[t]{2}{*}{$p$ - value } \\
\hline & & & \\
\hline Age, yrs $($ mean $\pm S D)$ & $72.3 \pm 13.2$ & $70.5 \pm 13.5$ & $<0.001$ \\
\hline$\%$ Female (n) & $61.4 \%(510)$ & $48.3 \%(964)$ & $<0.001$ \\
\hline \multicolumn{4}{|l|}{ Race - \% (n) } \\
\hline White & $31.9(422)$ & $29.3(547)$ & \multirow[t]{4}{*}{0.09} \\
\hline Black & $29.4(388)$ & $30.3(565)$ & \\
\hline Hispanic & $31.7(419)$ & $31.2(582)$ & \\
\hline Other/Unknown & $7.0(92)$ & $9.2(183)$ & \\
\hline GFR, mL/min (mean $\pm \mathrm{SD})$ & $42.8 \pm 13.1$ & $34.8 \pm 16.5$ & $<0.001$ \\
\hline Warfarin $-\%(n)$ & $61.5(813)$ & $52.0(970)$ & $<0.001$ \\
\hline Charlson Score (median $\pm \mathrm{SD})$ & $3.1 \pm 2.5$ & $3.7 \pm 2.5$ & $<0.001$ \\
\hline INR $($ median \pm SD) & $2.6 \pm 2.2$ & $2.8 \pm 2.5$ & $<0.001$ \\
\hline Platelet count, $\times 10^{9} / \mathrm{L}($ mean \pm SD $)$ & $188 \pm 80$ & $162 \pm 80$ & $<0.001$ \\
\hline History of bleeding $\%(\mathrm{n})$ & $3.2(42)$ & $2.4(44)$ & 0.16 \\
\hline \multicolumn{4}{|l|}{ Health Insurance \% (n) } \\
\hline Medicaid & $18.9(250)$ & $20.4(380)$ & \multirow[t]{4}{*}{0.81} \\
\hline Medicare & $69.6(920)$ & $67.7(1263)$ & \\
\hline Commercial & $11.2(148)$ & $11.7(219)$ & \\
\hline Self & $0.2(2)$ & $0.1(2)$ & \\
\hline
\end{tabular}

There was no difference in the groups in their history of prior bleeding episodes.

Incidence of Major Bleeding. A total of 80 episodes of major bleeding were observed within 10 days of the initiation of anticoagulation. The most common types of bleeding found in our cohorts were gastrointestinal, intra-abdominal and intrathoracic bleeds. Patients with GFR $<60 \mathrm{~mL} / \mathrm{min}$ treated with dalteparin were significantly less likely to experience a major bleed than patients treated with unfractionated heparin $(1.14 \%$ vs. $3.49 \%, p<0.001$, Fig. 1). Figures 2 and 3 represent bleeding episodes in subgroups defined by GFR 30-60 mL/ $\mathrm{min}$ and GFR $<30 \mathrm{~mL} / \mathrm{min}$ respectively. Figure 1 also demonstrates that the difference in the incidence of major bleeds occurs, as expected, generally within the first 10 days for both groups.

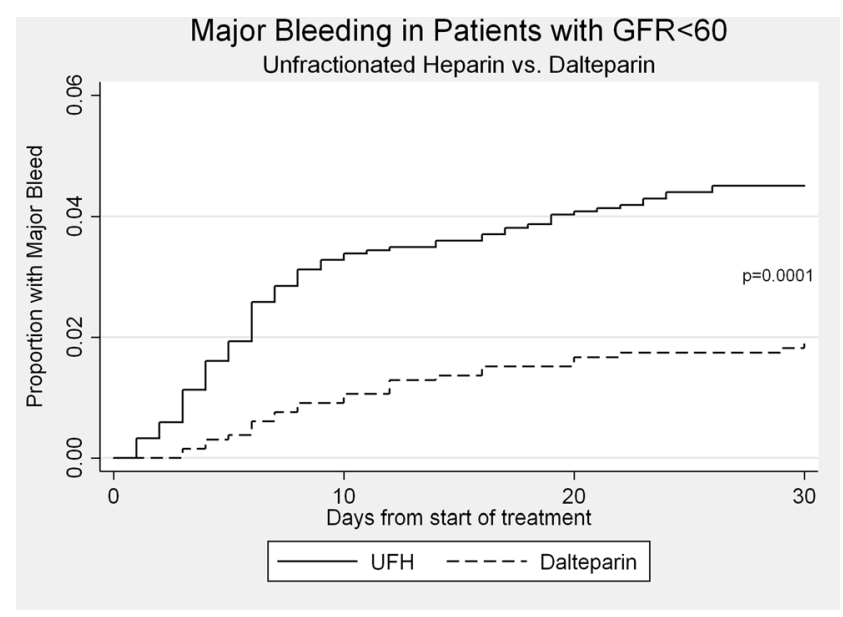

Figure 1. Major bleeding events for patients treated with unfractionated heparin vs. dalteparin.
Hazard Ratio Associated with Dalteparin Use. We examined the risk of bleeding associated with dalteparin therapy. Dalteparin use was associated with more than a $60 \%$ reduced risk that remained strong and significant after adjusting for age, sex, Charlson score, history of bleeding, use of warfarin, platelet count, GFR and INR (HR 0.39, $95 \%$ CI: $0.21-0.70)$, as demonstrated in Table 2. To determine if the degree of superiority of dalteparin over heparin in safety was similar among patients with higher vs. lower GFR, we performed a separate (stratified analysis) for patients with GFR $<30 \mathrm{~mL} / \mathrm{min}$ and patients with GFR between 30 and $60 \mathrm{~mL} / \mathrm{min}$. Among 927 patients with GFR $<30 \mathrm{~mL} / \mathrm{min}$, $215(23.2 \%)$ were treated with dalteparin and $712(76.8 \%)$ were treated with unfractionated heparin. Treatment with dalteparin was still associated with a lower likelihood of bleeding compared to treatment with unfractionated heparin, but the difference was no longer significant (HR 0.35, $95 \%$

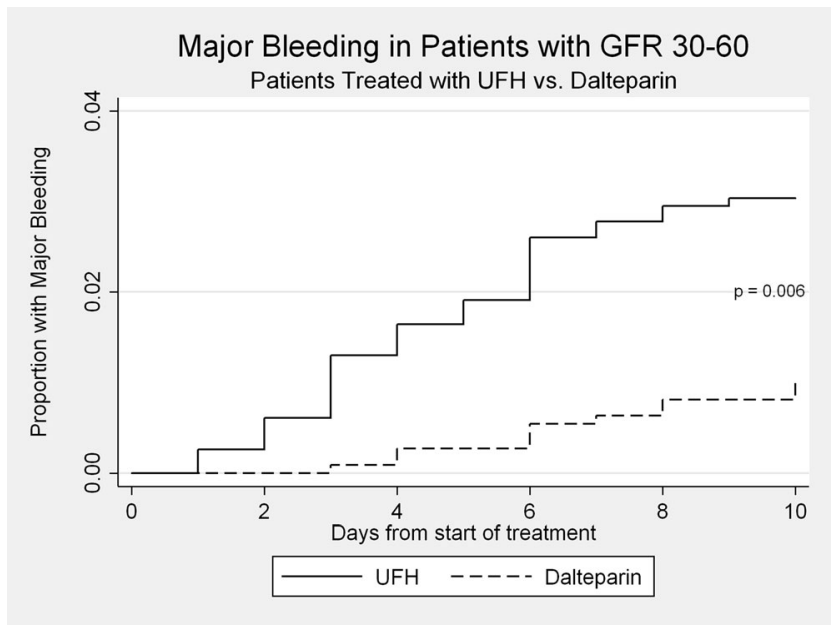

Figure 2. Major bleeding events for subgroup with GFR 30-60mL/ min, censored at 10 days. 


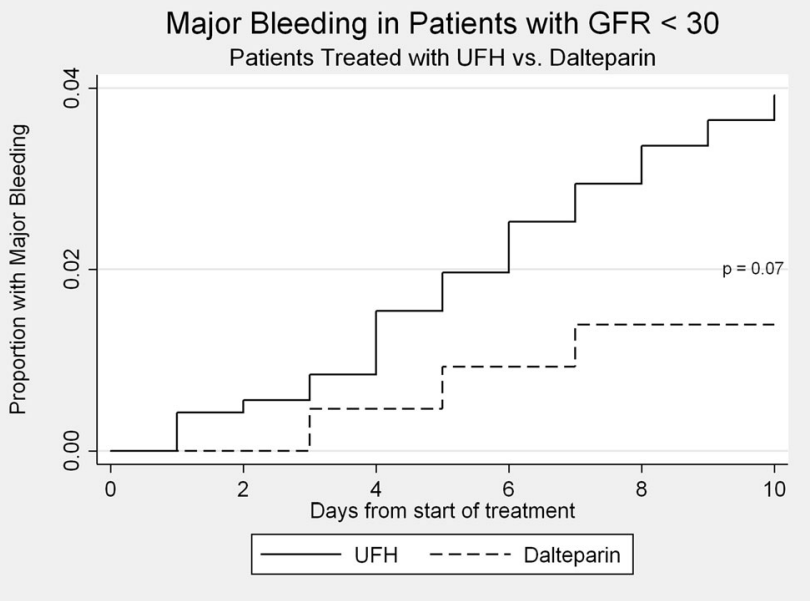

Figure 3. Major bleeding events for subgroup with GFR $<30 \mathrm{~mL} / \mathrm{min}$, censored at 10 days.

CI: 0.11-1.15), even after adjusting for patient characteristics (HR 0.37, $95 \%$ CI: 0.11-1.22). Among the 2,259 patients with GFR between 30 and $60 \mathrm{~mL} / \mathrm{min}, 1,106(49 \%)$ were treated with dalteparin and 1,153 (51\%) were treated with unfractionated heparin. Treatment with dalteparin was associated with a significantly lower risk of bleeding compared to treatment with unfractionated heparin (HR 0.32, $95 \%$ CI 0.16-0.64). This decrease in bleeding rate remained strong and significant after adjustments for the variables (HR 0.42, $95 \%$ CI 0.21-0.84). These data are also shown in Table 2.

\section{DISCUSSION}

In this large retrospective analysis, we demonstrate that treatment with therapeutic dose of dalteparin was associated with a significantly reduced risk of major bleeding in patients with renal insufficiency, compared to therapy with unfractionated heparin. Furthermore, our study confirms the superior safety profile of dalteparin in patients with mild CKD, and we suggest that the safety profile is maintained in those with severe renal insufficiency.

To our knowledge, this study is the first study that compares bleeding risks of therapeutic dalteparin and unfractionated heparin in hospitalized patients with renal insufficiency. The results of this study suggest that dalteparin is not inferior, and may be superior in safety to unfractionated heparin when used at therapeutic dosages in patients with a moderate to severe degree of renal impairment.

There have been both single dose and multi-dose studies with enoxaparin that raise concern for bioaccumulation of LMWHs. Becker et al. showed that therapeutic doses of enoxaparin given to patients with significant chronic renal impairment $(\mathrm{CrCl}<40 \mathrm{~mL} / \mathrm{min})$ resulted in higher plasma anti-factor Xa levels as well as a higher incidence of major bleeding outcomes. ${ }^{14}$ DeCarolis et al. reported a greater incidence of major bleeding in those with renal impairment who received therapeutic enoxaparin compared to healthy volunteers who received the same drug. ${ }^{15}$ Chow et al. also found a tight linear relationship between creatinine clearance and antiXa concentrations with enoxaparin. ${ }^{16}$ In contrast, TrujilloSantos et al. showed no difference in fatal bleeding between the two groups and reported a twofold mortality increase in the group receiving UFH versus those receiving any LMWH with $\mathrm{CrCl}$ less than $30 \mathrm{~mL} / \mathrm{min} .{ }^{17}$ In this study, information on the specific LMWHs used was not available. ${ }^{17}$ We propose that enoxaparin and dalteparin have different safety profiles in CKD.

It is well established that there are significant differences between the different LMWHs. There have been several animal model studies that have highlighted the unique pharmacokinetics of dalteparin that serve as the biological grounding for our favorable results in CKD patients. ${ }^{18}$ It has been postulated that the mechanisms of heparin catabolism consist of a balance between a saturable elimination via the reticuloendothelial system and the non-saturable renal tubular excretion. ${ }^{18-20}$ Various clearance studies in animal models and clinical settings have shown that the higher LMWHs such as tinzaparin $(6,500 \mathrm{Da})$ and dalteparin $(5,700 \mathrm{Da})$ have a faster, dose dependent clearance, suggesting greater involvement of the endothelial clearance pathway with increasing molecular weights and less renal clearance dependency. ${ }^{21,22}$

Rabbat and his group reported all patients who experienced bleeding on dalteparin thromboprophylaxis had trough anti-X a levels below the detection threshold. ${ }^{23}$

While bioaccumulation of enoxaparin in CKD had been established in aforementioned studies, further review of the literature reveals that the presumed clinical relationship of increased bleeding with renal insufficiency for enoxaparin has not been shown to be straightforward. Sharif-Askari

Table 2. Bleeding with Dalteparin vs. Unfractioned Heparin. Adjusted and Unadjusted Rates for all CRI Patients and for Patients Separated by GFR

\begin{tabular}{llll}
\hline \hline & $\begin{array}{l}\text { All Patients } \\
(\boldsymbol{n}=\mathbf{3 1 8 6})\end{array}$ & $\begin{array}{l}\text { GFR<30 mL/min } \\
(\boldsymbol{n}=\mathbf{9 2 7})\end{array}$ & $\begin{array}{l}\text { GFR 30-60 mL/min } \\
(\boldsymbol{n}=\mathbf{2 2 5 9})\end{array}$ \\
\hline Unadjusted HR* & 0.31 & 0.35 & 0.32 \\
(95\% CI) & $(0.17-0.55)$ & $(0.11-1.15)$ & $(0.16-0.64)$ \\
Adjusted HR* & 0.39 & 0.37 & 0.42 \\
(95\% CI) & $(0.21-0.70)$ & $(0.11-1.22)$ & $(0.21-0.84)$ \\
\hline
\end{tabular}

Adjusted for: age, sex, Charlson score, history of bleeding, use of warfarin, platelet count, GFR and INR

*Cox-proportional hazards model with major bleeding censored at 10 days after start of treatment 
et al. compared patients with CKD stages 3-5 and noted a higher incidence of major bleeding with enoxaparin over UFH (HR4.79). ${ }^{24}$ Thorevska and colleagues also reported an increased incidence of bleeding $(2.54 \mathrm{x})$ with therapeutic enoxaparin as compared to $\mathrm{UFH}^{25}$ in patients with GFR < $60 \mathrm{~mL} / \mathrm{min}$. Levine et al. showed a positive relationship between anti-factor Xa level and incidence of wound hematoma in hip replacement patients with once-daily prophylactic enoxaparin, ${ }^{26}$ whereas Sanderlink et al. showed that, after 5 days of enoxaparin prophylaxis, anti-factor $\mathrm{Xa}$ levels were found to be unrelated to renal function until $\mathrm{CrCl}<20 \mathrm{~mL} /$ $\min ^{27}$

Since there was a significant difference in the GFR in the treatment groups, we stratified according to GFR for those with GFRs $<30 \mathrm{~mL} / \mathrm{min}$ and those with 30-60 mL/min. Our study shows that the odds of bleeding for dalteparin found in our GFR $<30 \mathrm{~mL} / \mathrm{min}$ subgroup analysis were not higher, and that there was a trend towards a decreased risk of bleeding. We did not analyze anti-Factor Xa levels, but the evidence for higher anti-factor Xa levels with therapeutic dalteparin leading to a higher frequency of clinical bleeding in patients with renal impairment is weak. Tincani showed that no bioaccumulation occurred after 6 days of dalteparin prophylaxis, irrespective of renal function without major bleeding events. ${ }^{28}$ Studies performed by both Schmid et al. and Shprecher et al. showed no increase in anti-factor Xa level in patients with renal impairment in prophylactic or therapeutic dosing, ${ }^{10,29}$ and a metaanalysis from 2006 notes that insufficient data exist to assess risk for major bleeding for other LMWHs such as dalteparin and tinzaparin, while still advising adjusted dose enoxaparin in $\mathrm{CKD}^{30}$

Strengths. Our study represents diverse, real-world situations where dalteparin would be used for varying indications and durations. The retrospective nature of our study allowed us to collect a much larger number of patients for each arm than had been possible for the prospective studies, and control for a large number of variables within each arm. Our outcome measurement was complemented by both quantitative (hemoglobin and units of blood required) and qualitative reports (event documentations).

Limitations. Limitations of this study include the nonrandomized, retrospective nature and its single center design. Relying on charts and their written progress notes and other documentations may have made missing some of the bleeding outcomes inevitable, but should not have biased one group's results over the other. Also, we acknowledge that we did not have a way of ensuring that we captured $100 \%$ of measurable bleeding events, as our hospital is not a closed system of care, but we do capture the vast majority of care in the region and it is likely that patients who were seen by our hospital system once will return to the same hospital system. Some major risk factors such as corticosteroid use and peptic ulcer disease were not accounted for. Residual confounding may remain due to the fact that using Charlson score may have underestimated the effect of individual comorbidities in this particularly sick population. Another unavoidable limitation was using absolute dose cutoffs to define therapeutic anticoagulation rather than weight-based dosing, so it is possible that we could have missed instances of inadvertent or conscious underdosing on the part of providers who were aware of their patients' CKD. The characteristics of our dalteparin and unfractionated groups were grossly similar, but had key differences, such as female predominance in the LMWH group; but, given that relatively higher anti factor Xa levels have been associated with LMWH in females, this again should not have biased the results significantly. ${ }^{28,31,32}$

In conclusion, contrary to previous studies that seemed to suggest a higher risk of bleeding associated with LMWH in those with renal insufficiency, our retrospective study showed that the LMWH dalteparin at therapeutic doses does not result in increased instances of clinically significant hemorrhage. Our findings serve as convincing evidence that increased safety concerns for dalteparin use may not be warranted, and therefore the ease of use and steady pharmacokinetics for LMWH may make this therapy preferred over the frequent monitoring of aPTT and the inherent flaws of unpredictable pharmacokinetics for UFH.

We suggest that dalteparin be used with greater confidence at therapeutic doses for patients in in-hospital settings with mild-to-moderate renal insufficiency.

Acknowledgements: We thank Eran Bellin $M D P h D$ and the CLG staff for help in data procurement.

Conflict of Interest: The authors declare that they do not have a conflict of interest.

Author Contributions: The authors are solely responsible for the views expressed in this article.

Funding: There was no external funding for this work.

Prior presentations: The findings of this paper were presented as a poster at the 36th Society of General Internal Medicine (SGIM) Annual Meeting on 24-27 April 2013 in Denver, Colorado.

Corresponding Author: Doyun Park, MD; Division of HematologyOncologyMount Sinai-Roosevelt Hospital, 1000 Tenth Avenue, New York, NY, USA (e-mail: dpark@chpnet.org).

\section{REFERENCES}

1. Wells PS, Forgie MA, Rodger MA. Treatment of venous thromboembolism. JAMA. 2014;311:717-728.

2. Alqiwaizani M, Buckley L, Adams C, Fanikos J. Anticoagulants: A review of the pharmacology, dosing, and complications. Curr Emerg Hosp Med Rep. 2013;21(1):83-97. 
3. Weitz JI. Low-molecular-weight heparins. N Engl J Med. 1997;337:688698.

4. Gouin-Thibault I, Pautas E, Sigueret V. Safety profile of different lowmolecular weight heparins used at therapeutic dose. Drug Saf. 2005;28:333-349.

5. Lee AY, Levine MN, Baker RI, et al. Low-molecular weight heparin versus a coumarin for the prevention of recurrent venous thromboembolism in patients with cancer. N Engl J Med. 2003;349:146-153.

6. Green B, Greenwood M, Saltissi D, et al. Dosing strategy for enoxaparin in patients with renal impairment presenting with acute coronary syndromes. Br J Clin Pharmacol. 2005;59:281-290.

7. Siguret V, Pautas E, Février M, et al. Elderly patients treated with tinzaparin (Innohep) administered once daily (175 anti-Xa IU/kg): anti-Xa and anti-IIa activities over 10 days. Thromb Haemost. 2000;84:800-804.

8. Pautas E, Gouin I, Bellot O, Andreux JP, Siguret V. Safety profile of tinzaparin administered once daily at a standard curative dose in two hundred very elderly patients. Drug Saf. 2002;25:725-733.

9. Spyropoulos AC, Mahan C. Venous thromboembolism prophylaxis in the medical patient: controversies and perspectives. Am J Med. 2009; 122: 1077-1084.

10. Shprecher AR, Cheng-Lai A, Madsen EM, et al. Peak antifactor xa activity produced by dalteparin treatment in patients with renal impairment compared with controls. Pharmacotherapy. 2005;25:817-822.

11. Holbrook A, Schulman S, Witt DM, et al. On behalf of the American College of Chest Physicians. Evidence-based management of anticoagulant therapy: Antithrombotic Therapy and Prevention of Thrombosis, 9th ed: American College of Chest Physicians Evidence-Based Clinical Practice Guidelines. Chest. 2012;141:e152S-e184S.

12. Fragmin (dalteparin) [product monograph]. Kirkland, Quebec, Canada: Pfizer Canada Inc; August 2014.

13. Schulman S. Kearon $\mathrm{C}$ on behalf of the subcommittee on control of anticoagulation of the Scientific and Standardization committee of the International Society on Thrombosis and Haemostasis. Definition of major bleeding in clinical investigations of antihemostatic medicinal products in non-surgical patients. Scientific and Standardization Committee Communication. J Thromb Haemost. 2005;3:692-694.

14. Becker RC, Spencer FA, Gibson M, et al. Influence of patient characteristics and renal function on factor Xa inhibition pharmacokinetics and pharmacodynamics after enoxaparin administration in non-ST-segment elevation acute coronary syndromes. Am Heart J. 2002;143:753-759.

15. DeCarolis DD, Thorson JG, Clairmont MA, Leuthner AM, Rector TS, Johnson GJ. Enoxaparin outcomes in patients with moderate renal impairment. Arch Intern Med. 2012;172:1713-1718.

16. Chow SL, Zammit $\mathbf{K}$, West $\mathbf{K}$, Dannenhoffer $\mathbf{M}$, Lopez-Candales A Correlation of antifactor Xa concentrations with renal function in patients on enoxaparin. J Clin Pharmacol. 2003;43:586-590.

17. Trujillo-Santos J, Schellong S, Falga C, et al. Low-molecular-weight or unfractionated heparin in venous thromboembolism: the influence of renal function. Am J Med. 2013;126:425-434.

18. Johansen KB, Balchen T. Tinzaparin and other low-molecular-weight heparins: what is the evidence for differential dependence on renal clearance? Exp Hematol Oncol. 2013;2:21.
19. Peyrou V, Lormeau JC, Caranobe C, et al. Pharmacological properties of CY 216 and of its ACLM and BCLM components in the rabbit. Thromb Haemost. 1994;72:268-274.

20. Palm M, Mattsson C. Pharmacokinetics of heparin and low molecular weight heparin fragment (Fragmin) in rabbits with impaired renal or metabolic clearance. Thromb Haemost. 1987;58:932-935.

21. Johansen KB, Schroeder M, Lundtorp L, Mousa SA. Renal elimination of tinzaparin versus enoxaparin in normal versus nephrectomized rats. J Thromb Haemost. 2007;5(Suppl 2):629.

22. Collignono F, Frydman A, Caplain H, et al. Comparison of the pharmacokinetic profiles of three low molecular mass heparin-dalteparin, enoxaparin and nadroparin-administered subcutaneously in healthy volunteers (doses for prevention of thromboembolism). Thromb Haemost. 1995;73:630-640

23. Rabbat CG, Cook DJ, Crowther MA, et al. Dalteparin thromboprophylaxis for critically ill medical-surgical patients with renal insufficiency. J Crit Care. 2005;20:357-363.

24. Saheb Sharif-Askari F, Syed Sulaiman SA, Saheb Sharif-Askari N, Al Sayed Hussain A, Railey MJ. Adverse outcomes of anticoagulant use among hospitalized patients with chronic kidney disease: a comparison of the rates of major bleeding events between unfractionated heparin and enoxaparin. PLoS One. 2014;9:e106517.

25. Thorevska N, Amoateng-Adjepong $\mathbf{Y}$, Sabahi R, et al. Anticoagulation in hospitalized patients with renal insufficiency: a comparison of bleeding rates with unfractionated heparin vs enoxaparin. Chest. 2004; 125:856863.

26. Levine MN, Planes A, Hirsh J, et al. The relationship between anti-factor Xa level and clinical outcome in patients receiving enoxaparin low molecular weight heparin to prevent deep vein thrombosis after hip replacement. Thromb Haemost. 1989;62:940-944.

27. Sanderink GJ, Guimart CG, Ozoux ML, Jariwala NU, Shukla UA Boutouyrie BX. Pharmacokinetics and pharmacodynamics of the prophylactic dose of enoxaparin once daily over 4 days in patients with renal impairment. Thromb Res. 2002;105:225-231.

28. Tincani E, Mannucci C, Casolari B, et al. Safety of dalteparin for the prophylaxis of venous thromboembolism in elderly medical patients with renal insufficiency: a pilot study. Haematologica. 2006;91:976-979.

29. Schmid P, Brodmann D, Fischer AG, Wuillemin WA. Study of bioaccumulation of dalteparin at a prophylactic dose in patients with various degrees of impaired renal function. J Thromb Haemost. 2009;7:552-558.

30. Lim W, Dentali F, Eikelboom JW, Crowther MA. Meta-analysis: lowmolecular-weight heparin and bleeding in patients with severe renal insufficiency. Ann Intern Med. 2006; 144:673-684.

31. Oldgren J, Johnston N, Siegbahn A. Xa inhibition and coagulation activity-the influence of prolonged dalteparin treatment and gender in patients with acute coronary syndrome and healthy individuals. Am Heart J. 2008;155:493.e1-8.

32. Dufour B, Toussaint-Hacquard M, Kearney-Schwartz A, et al. Glomerular filtration rate estimated by Cockcroft-Gault formula better predicts anti-Xa levels than modification of the diet in renal disease equation in older patients with prophylactic enoxaparin. J Nutr Health Aging. 2012;16:647-652 